\title{
Increased expression of multidrug resistance related proteins Pgp, MRP1, and LRP/MVP occurs early in colorectal carcinogenesis
}

\author{
G A Meijer, A B Schroeijers, M J Flens, S G M Meuwissen, P van der Valk, J P A Baak, \\ R J Scheper
}

\begin{abstract}
Aim-To analyse the expression of multidrug resistance (MDR) related proteins at different steps in colorectal carcinogenesis.

Methods-The presence of three MDR related proteins (Pgp, MRP1, and LRPI MVP) was studied by means of immunohistochemistry in normal, adenomatous, and malignant colorectal epithelium. Formaldehyde fixed, paraffin embedded tissue sections of 17 samples of colorectal tissue were used (normal mucosa, $n=4$; adjacent mucosa, $n=5$; adenoma, $n=5$; carcinoma, $n=3$ ).

Results-For all three proteins, expression was found in the surface epithelium and the upper parts of the crypts in normal colon. In the adenomas, staining was seen along the complete length of the crypts. In the carcinomas analysed, all epithelium showed positive staining. Mucosa adjacent to either carcinoma or adenoma showed staining patterns mostly resembling those of normal mucosa, but sometimes some extension of staining was seen along the crypt.

Conclusions-These proteins already show increased expression in the adenoma stage. In the absence of adequate mucin production in adenomas, MDR related proteins could be an important factor in protecting the epithelium against further environmentally induced genetic damage. This could be one of the reasons why only about $5 \%$ of colorectal adenomas will actually progress to carcinomas.

(F Clin Pathol 1999;52:450-454)
\end{abstract}

Keywords: multidrug resistance; colon; carcinogenesis

Colorectal cancer arises from normal intestinal epithelium through various genetic changes, each contributing to oncogenesis. Dietary factors that determine the environment of the intestinal epithelium are thought to play an important role in this process. Agents entering the mucosal cells from the stools can cause genetic damage, thereby inducing progression of the epithelium along the neoplastic pathway. ${ }^{1}$ In normal colonic mucosa defence mechanisms are presumably active to eliminate such toxic agents, or xenobiotics, from the cell.

Several mechanisms providing protection against toxic agents (notably cytotoxic drugs) have also been described in tumour cells.
Tumour cells often show broad cross resistance between unrelated classes of cytotoxic drugs. This phenomenon is known as multidrug resistance (MDR). ${ }^{2}$ To date, at least two multidrug resistance mechanisms have been unravelled, involving members of the ATP binding cassette transporter superfamily, namely $\mathrm{P}$ glycoprotein (Pgp) and the multidrug resistance related protein (MRP1).

Pgp and MRP1 function as transmembrane drug efflux pumps, decreasing intracellular drug accumulation, thereby conferring resistance to various natural product drugs..$^{3-7}$ LRP/ MVP, which stands for "lung resistance related protein/major vault protein," is another multidrug resistance related protein that was discovered in a non-small-cell lung cancer cell line, selected for doxorubicin resistance. ${ }^{8}$ The LRP/ MVP gene was cloned and identified as the human major vault protein. ${ }^{9}$ Vaults are recently described cellular organelles broadly distributed and highly conserved among diverse eukaryotic cells. ${ }^{10}$ Although different from ATP binding cassette molecules, vaults have also been implicated in transport of various substrates, including cytotoxic drugs. ${ }^{11-13}$ These multidrug resistance related proteins (Pgp, MRP1, and LRP/MVP) are broadly distributed in normal cells. ${ }^{14-16}$ High levels of Pgp have been localised to the apical surfaces of small and large intestinal epithelium. ${ }^{17-19}$ MRP1 and LRP/MVP expression has also been found in the digestive tract. ${ }^{14}{ }^{16}$ Furthermore, frequent Pgp, MRP1, and LRP/MVP expression was found in cancers derived from mucosal tissues, such as the colon.

Although the physiological function of the multidrug resistance related proteins in the various human tissues is unknown, the distribution and combined expression in distinct human cells implies a function in protection of the cells against xenobiotics. Our aim in the present study was to analyse the expression of multidrug resistance related proteins at different steps in colorectal carcinogenesis. To this end we determined the presence of Pgp, MRP1, and LRP/MVP by immunohistochemistry in normal colorectal epithelium, colorectal adenomas, colorectal carcinomas, and colorectal mucosa adjacent to adenomas or carcinomas, using a panel of monoclonal antibodies (MAb) that we have developed to the multidrug resistance proteins Pgp, LRP/MVP, and MRP1. 


\section{Methods}

Formaldehyde fixed, paraffin embedded $4 \mu \mathrm{m}$ tissue sections of 17 samples of colorectal tissue (normal mucosa from resection margins of colectomy specimens further referred to as normal mucosa, $n=4$; normal mucosa adjacent to adenoma or carcinoma further referred to as adjacent mucosa, $\mathrm{n}=5$; adenoma, $\mathrm{n}=5$; carcinoma, $\mathrm{n}=3$ ) were obtained from the tissue bank of the department of pathology of the Free University Hospital. The monoclonal antibodies JSB- $1^{20}$ and $4 \mathrm{E} 3^{21}$ raised against Pgp, MRPr1 and MRPm6 raised against MRP1, ${ }^{22}$ and LRP-56 raised against $\mathrm{LRP} / \mathrm{MVP}^{8}$ were used. All antibodies are murine except for the rat MAb MRPr1.

The formaldehyde fixed, paraffin embedded tissue sections were deparaffinised in xylene, rehydrated, and endogenous peroxidase activity was blocked by $0.3 \%$ (vol $/ \mathrm{vol}$ ) $\mathrm{H}_{2} \mathrm{O}_{2}$ in methanol for 30 minutes. For staining with JSB-1, 4E3, and MRPm6, paraffin sections were pretreated with citrate buffer $(0.01 \mathrm{M}$ citric acid ( $\mathrm{pH} \mathrm{6.0)}$ in distilled water) for $3 \times$ five minutes at $100^{\circ} \mathrm{C}$ in a microwave oven. Slides were blocked with normal goat or rabbit serum for 20 minutes and incubated with JSB-1 (1-4 $\mu \mathrm{g} / \mathrm{ml}), 4 \mathrm{E} 3 \quad(5-10 \mu \mathrm{g} / \mathrm{ml}), \operatorname{MRPr} 1 \quad(10-20$ $\mu \mathrm{g} / \mathrm{ml})$, MRPm6 $(20-40 \mu \mathrm{g} / \mathrm{ml})$, and LRP-56 $(5-10 \mu \mathrm{g} / \mathrm{ml})$ at $4^{\circ} \mathrm{C}$ overnight. All reagents were diluted in phosphate buffered saline containing $1 \%(\mathrm{wt} / \mathrm{vol})$ bovine serum albumin (PBS/BSA). A biotin-streptavidin immunoperoxidase (ABC) method was applied using biotinylated goat anti-rat IgG (1:100, Jackson Immmunoresearch Laboratories) or rabbit anti-mouse IgG F(ab')2 (1:500, Dako) and streptavidin conjugated to horseradish peroxidase (1:500, Zymed). Bound peroxidase was developed with $4 \mathrm{mg}$ (wt/vol) 3,3'diaminobenzidine tetrahydrochloride (Sigma) and $0.02 \%$ (vol/vol) $\mathrm{H}_{2} \mathrm{O}_{2}$ in $100 \mathrm{ml} \mathrm{PBS}$; or 4 $\mathrm{mg} \mathrm{(wt/vol)} \mathrm{3-amino-9-ethylcarbazole} \mathrm{and}$ $0.02 \%$ (vol $/ \mathrm{vol}$ ) $\mathrm{H}_{2} \mathrm{O}_{2}$ in $0.1 \mathrm{M} \mathrm{NaAc}$ $(\mathrm{pH}=5.0)$. All slides were counterstained with haematoxylin and mounted.

Positive controls for Pgp, MRP, 1 and LRP/MVP staining included $4 \mu \mathrm{m}$ sections from formaldehyde fixed, paraffin embedded tissue of histological normal adrenal gland and lung. In addition, we used three doxorubicin selected multidrug resistance tumour cell lines, which were formaldehyde fixed and paraffin embedded like the tissues ${ }^{23}$ : SW-1573/2R160 (non-small-cell lung cancer; Pgp positive), GLC4/ADR (small cell lung cancer; MRP1 positive), and SW-1573/2R120 (LRP/MVP positive).

Negative controls included both omission of the first steps and substitution of the primary antibodies by different non-reactive antibodies of the same origin and isotype. Immunohistochemical expression of the multidrug resistance related proteins was evaluated and agreed upon by three observers, on the basis of intensity of staining, cellular distribution, and especially distribution in the colonic crypts.

\section{Results}

In the normal colorectal epithelium, the Pgp antibody 4E3 showed strong cytoplasmic membrane staining at the surface epithelium and the very upper part of the crypts (fig 1A). Occasionally, at this localisation some diffuse cytoplasmic staining was also found. In the adenomas, cytoplasmic membrane staining was observed along the complete length of the crypts (fig 1B). The cytoplasmic staining was more pronounced than in the normal epithelium. In the carcinomas an intense cytoplasmic membrane staining was seen predominantly, with diffuse cytoplasmic staining being even more intense than in the adenomas (fig 1C). It was notable that mucosa adjacent to either carcinoma or adenoma showed staining patterns mostly resembling those of normal mucosa, but occasionally some extension of staining was seen along the crypt, as in adenomas. A similar pattern of staining for Pgp in the respective colorectal epitheliums was found with JSB-1.

In the normal epithelium, staining of MRP1 was observed in the apical side of the cytoplasm, just below the level stained with the Pgp MAbs. The brush border and goblet cells were completely negative, as reported before. ${ }^{16}$ Also for MRP1, surface epithelium and upper parts of the crypts were clearly positive whereas in the lower three quarters of the crypts only some basal cytoplasmic staining could occasionally be seen (fig 1D).

In the adenomas, clear cytoplasmic staining was seen for all cells throughout crypts, which was finely granular (fig $1 \mathrm{E}$ ).

In the carcinomas, MRP1 staining was even more intense than in the adenomas and showed a more coarsely granular, apical cytoplasmic staining (fig $1 \mathrm{~F}$ ). Furthermore, the staining pattern of adjacent mucosa mostly resembled that of normal mucosa with occasionally some extension of staining along the crypt. As reported before, the staining intensity of MRPm6 was somewhat weaker than that of MRPr1, but similar staining patterns were observed. ${ }^{16}$

As detected by LRP-56, the distribution of LRP/MVP in the colorectal mucosa was, like MRP1, predominantly granular in the outer zone of the cytoplasm, just below the Pgp staining. Strong cytoplasmic staining was seen in the surface epithelium and upper parts of the crypts in normal epithelium (fig 1G), compared with diffuse cytoplasmic staining of the whole crypt in adenomas (fig $1 \mathrm{H}$ ). This staining pattern was also observed in the cytoplasm of carcinomas (fig 1I). The distribution of staining in adjacent mucosa was similar to that found for MRP1.

In colorectal adenomas an abrupt change from substantially normal epithelium to adenomatous epithelium could occasionally be found within a single crypt. ${ }^{24}$ This allows one to observe within one crypt the contrasting levels of the multidrug resistance related proteins between neoplastic and non-neoplastic epithelium. Low levels of Pgp, MRP1, and LRP/MVP were observed in the non-neoplastic epithelium. Precisely where stratification of nuclei indicates the start of neoplastic change, a definite increase of 
multidrug resistance related protein levels was seen (fig $1 \mathrm{~J}-\mathrm{L}$ ).

\section{Discussion}

There is much evidence to support the view that mutagenic agents in the environment of the colorectal epithelium - that is, the luminal contents of the gut-play a major role in the aetiology of colorectal cancer. Bile acids, mutagens formed by frying of fat and meat, and bacteria produced mutagens have all been associated with colorectal carcinogenesis. ${ }^{25-27}$ It is most likely that in the normal colonic mucosa protection mechanisms are active to eliminate these toxic agents, or xenobiotics, from the cell into the lumen. Since multidrug resistance related proteins have been shown to be physiologically present in the normal colorectal epithelium they are good candidates for involvement in this detoxification process. Pgp is prominently expressed in the apical cytoplasmic membranes of organs with excretory functions. ${ }^{17}{ }^{18}$ Recently, in colon cell lines the efflux of benzo[a]pyrene, a dietary carcinogen, was shown to be dependent on the activity of Pgp, indicating a possible role of Pgp in the
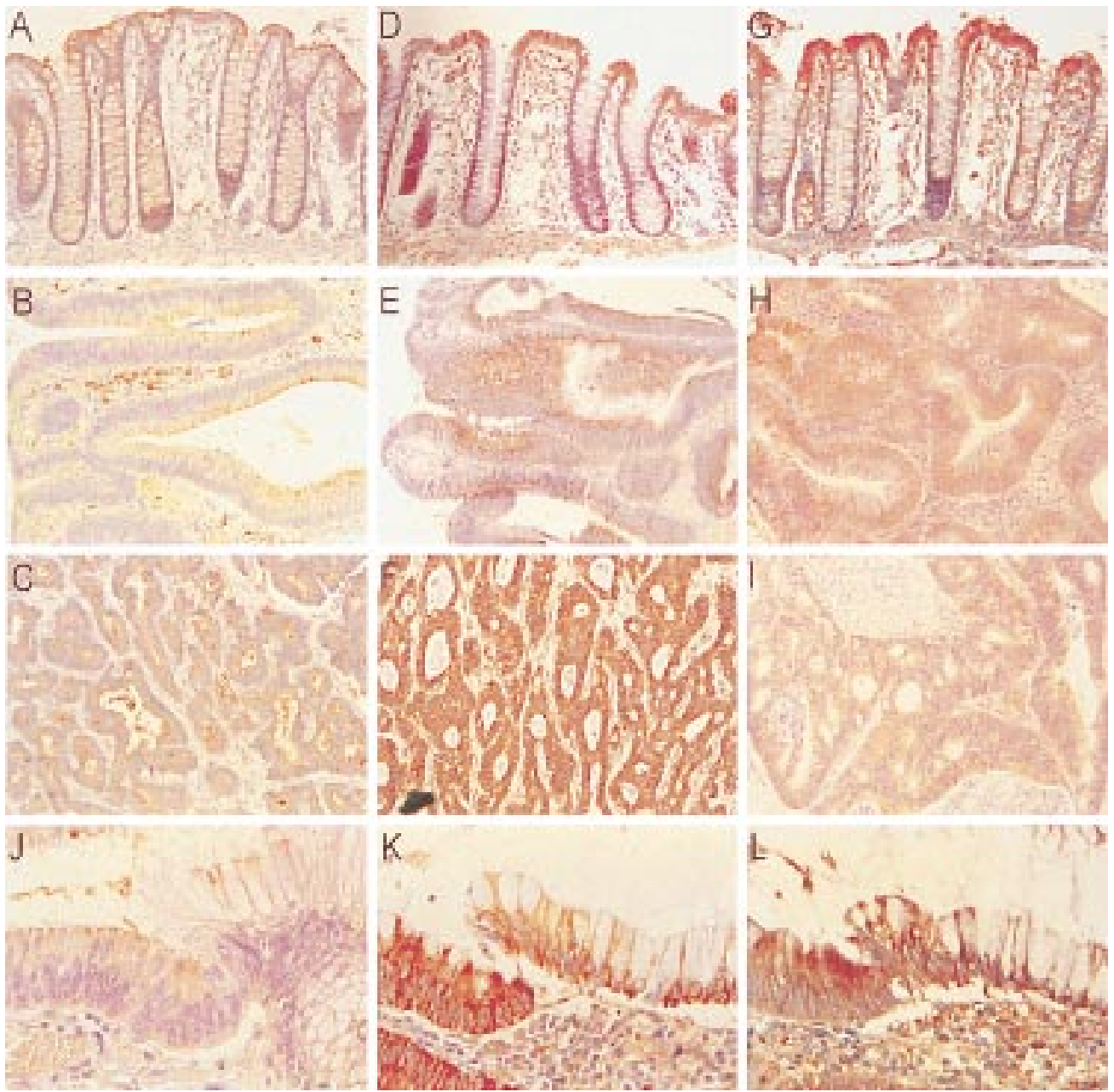

Figure 1 (A) Pgp staining in paraffin sections of normal colorectal epithelium with the 4E3 monoclonal antibody shows strong membrane staining at the surface epithelium and the very upper part of the crypts, with occasional slight cytoplasmic staining. (B) Adenomas show membrane staining along the complete length of the crypts with stronger cytoplasmic staining than in the normal epithelium. (C) Carcinomas show intense membrane staining, and the diffuse cytoplasmic staining was even more intense than in the adenomas. (D) MRP1 of normal colorectal epithelium with the MRPr1 monoclonal antibody shows apical cytoplasmic staining, with the brush border and goblet cells completely negative. The surface epithelium and upper parts of the crypts are clearly positive whereas in the lower three quarters of the crypts only some basal cytoplasmic staining can occasionally be seen. (E) In the adenomas, a clear, fine granular cytoplasmic staining is seen along the whole length of the crypts. $(F)$ In the carcinomas, staining with MRPr1 was even more pronounced than in adenomas and showed a coarsely granular cytoplasmic pattern with more intense staining near the plasma membrane. (G) LRP/MVP staining of normal colorectal epithelium with the LRP-56 monoclonal antibody shows strong cytoplasmic signals in the surface epithelium and upper parts of the crypts. (H) Diffuse cytoplasmic staining of the whole crypt is seen in adenomas.

(I) Strong cytoplasmic staining occurs for the carcinomas. $((A),(D),(G)$ : Surface of the normal epithelium is at the top of the figure; $(B),(E),(H)$ : Surface of the adenomas is at the left of the figure. In adenomas the crypts are longer and more irregular; (C), (F), (I): Only central parts of the carcinomas are shown, no surface visible. Panels (A)-(I), $\times 20$ objective).

(F) High power view of the 4E3 staining for Pgp in an adenomatous crypt with an abrupt change from substantially normal epithelium to neoplastic epithelium. In the non-neoplastic epithelium (right) no staining of the goblet cells and intense cytoplasmis membrane staining of the absorptive cells is seen. In the adenomatous epithelium (left) diffuse cytoplasmic staining is seen. The membrane staining of $4 E 3$ also seen in adenomas is not very clear in this area. (K) For MRP1 stained with MRPr1, predominantly basal staining is seen in the normal epithelium (right), with slightly stained absorptive cells in between the goblet cells. Precisely where stratification of nuclei indicates the start of neoplastic change (left), a definite increase in MRP1 expression is seen. (Ll) The same holds true for LRP/MVP detected with LRP-56. (Panels (f) $-(L), \times 63$ objective.) 
protection of colorectal epithelium against carcinogenic agents. ${ }^{28}$ In both a model system of mature rat enterocytes and in vivo in humans, the activity of mdrl-the gene coding for Pgp - was inducible by several compounds including commonly used oral antibiotics. ${ }^{29}$ MRP1 and LRP/MVP staining is different from the predominantly membrane staining observed for Pgp. The granular cytoplasmic staining pattern of MRP1 and LRP/MVP in colonic epithelium suggests a role for these proteins in the transport of compounds into intracellular compartments. An additional complication is the possibility that transporters are differentially rerouted in the cell. ${ }^{30}$ Evers et al found transport of a glutathione-S-conjugate and daunorubicin in a human MRP1 transfected kidney cell line. In these epithelial cells MRP1 is localised mainly to the basolateral plasma membrane, unlike Pgp which is localised more apically. ${ }^{31}$ The physiological role of MRP1 and its recently identified homologues cMOAT and MRP3-6 is still unknown. ${ }^{32}$ In vivo studies showed that MRP1 can act as a pump for drugs conjugated to glutathione (GSH), or glucuronides. ${ }^{33-35}$ Alternatively, drug efflux by MRP1 could occur by a cotransport mechanism involving reduced GSH and possibly other anions. ${ }^{36}$

The physiological function of LRP/MVP also remains to be resolved. Although different from ATP binding cassette molecules like Pgp and MRP1, the major vault protein LRP/MVP has also been implicated in transmembrane transport of various substrates. ${ }^{11}{ }^{12}$ The observation that vault synthesis is linked directly to multidrug resistance supports a direct role for vaults in drug resistance. ${ }^{13}$ Direct proof that vaults are actually able to transport cytostatic drugs, however, is still lacking. Clinical interest in LRP/MVP relates to recent studies in which LRP/MVP expression was predictive of poor response to chemotherapy. In normal human tissues LRP/MVP expression showed distinct

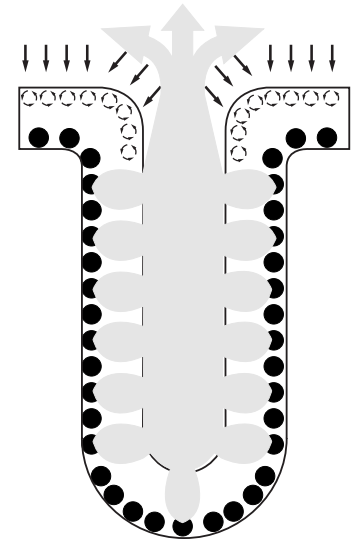

Normal mucosa

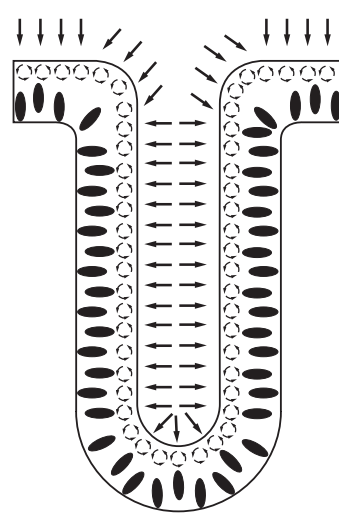

Adenoma

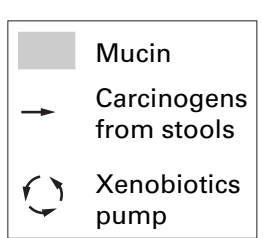

Figure 2 Increased expression of the multidrug resistance related proteins Pgp, MRP1, and $L R P / M V P$ in adenomas could be secondary to mucin depletion. In normal colorectal mucosa (left), the continuous production and flow of mucin by goblet cells protects especially the deeper parts of the crypts from carcinogenic agents from the stools. Only at the surface and in the upper parts of the crypts are xenobiotic pumps active. In the adenomatous (neoplastic) crypts (right), the lack of mucin facilitates a higher concentration of xenobiotics in the deeper parts of the crypts and as a result the expression of xenobiotic pumps is induced to levels normally only seen at the surface epithelium. patterns suggesting a role in detoxification processes, similar to Pgp and MRP $1 .{ }^{14}$

It could be hypothesised that a disturbance of the balance between attack and defence mechanisms - for example, by impaired functioning of xenobiotics pumps - could be an initiating step in the pathogenesis of colorectal cancer. One then would expect a decreased expression of these proteins in the precursor lesion of colorectal cancer, the adenoma. However, this is clearly not what we found for Pgp, MRP1, and LRP/MVP. On the contrary, adenomas already showed increased expression of these proteins, as detected by the monoclonal antibodies in our present study. This increased expression of Pgp, MRP1, and LRP/ MVP occurs early during oncogenesis and appeared to be directly related to the change from non-neoplastic to neoplastic epithelium (fig 1, J-L).

Increased expression of these multidrug resistance related proteins could be explained in several ways. First, genetic changes underlying the neoplastic phenotype could also cause the increased expression of xenobiotic pumps. Two closely related genes, MDR 1 and MDR 3 , which encode highly homologous $\mathrm{P}$ glycoproteins in humans, are both located at the long arm of chromosome 7. Indeed, extra copies of chromosome 7 have often been observed in colorectal adenomas. ${ }^{37}$ However, gain of the short arm of chromosome 16, where the genes for MRP1 and LRP/MVP are located, was reported in only two of 23 colorectal tumours (nine adenomas and 14 carcinomas). ${ }^{938}{ }^{39}$ For most colorectal tumours it is therefore unlikely that the increased expression of MRP1 and LRP/MVP can be explained in this way.

Second, increased expression of Pgp, MRP1, and LRP/MVP could be a non-specific feature of impaired differentiation, which is a feature of neoplastic epithelium. This, however, does not fit very well with the observation that Pgp expression was less in poorly differentiated colorectal cancers than in well differentiated tumours. ${ }^{19}$ MRP1 expression has also been negatively correlated with differentiation in lung cancers. ${ }^{40}$

A more plausible explanation is that the increased expression of xenobiotic pumps occurs secondary to the failure of some other defence mechanism. One of the main protective mechanisms present in the colorectal epithelium is the continuous production and flow of mucin by goblet cells. One of the features of neoplastic (or dysplastic) colorectal epithelium is lack of mucin. ${ }^{41}$ The lack of mucin may facilitate a higher concentration of xenobiotics in the deeper parts of the crypts and as a result the expression of multidrug resistance related proteins may be induced to levels normally only seen at the surface epithelium (fig 2).

Colorectal cancer is regarded to be intrinsically resistant to chemotherapy - that is, resistant to chemotherapy at the time of diagnosis, a feature shared with other cancers associated with an increased exposure to carcinogens like small cell lung cancer. This resistant phenotype 
fits with the findings of our present study, showing increased expression of Pgp, MRP1, and LRP/MVP in adenomas which are precursors of colorectal cancers. This observation may also have implications for chemoprevention, which aims at preventing the development of colorectal cancers by dietary modifications or supplementation with agents believed to reduce the risk of such cancers. ${ }^{42}$ Possibly certain chemopreventive agents may not reach adequate concentrations in the epithelium of adenomas because they are rapidly cleared from the cytoplasm by xenobiotic pumps. Thus certain chemopreventive agents might be less effective once an adenoma has occurred.

Multidrug resistance related proteins are likely to be players in the field of interaction between environmental agents and cell biological defence mechanisms in the colonic mucosa. The results of the present study indicate that some of these proteins already show increased expression in adenomas. In the absence of adequate mucin production in adenomas, multidrug resistance related proteins could be an important factor in protecting the epithelium against further environmentally induced genetic damage (fig 2). This could be one of the reasons why only about $5 \%$ of colorectal adenomas actually progress to carcinomas.

Supported by grant No 28-1687 of the Praeventiefonds and KWF-VU95-923.

1 Potter JD. Colon cancer-do the nutritional epidemiology, the gut physiology and the molecular biology tell the same story? F Nutr 1993;123:418-423.

2 Biedler JL. Genetic aspects of multidrug resistance. Cancer 1992;70:1799-809

3 Higgins CF. ABC transporters-from microorganisms to man. Annu Rev Cell Biol 1992;8:67-113.

4 Endicott JA, Ling V. The biochemistry of P-glycoproteinmediated multidrug resistance. Annu Rev Biochem 1989;58: mediated $137-71$.

5 Cole SPC, Bhardwaj G, Gerlach JH, et al. Overexpression of a transporter gene in a multi-drug resistant human lung cancer cell line. Science 1992;258:1650-4.

6 Gottesman MM, Pastan I. Biochemistry of multidrug resistance mediated by the multidrug transporter. Annu Rev Biochem 1993;62:385-427.

7 Zaman GJR, Flens MJ, van Leusden MR, et al. The human multidrug resistance-associated protein MRP is a plasma membrane drug-efflux pump. Proc Natl Acad Sci USA 1994;91:8822-6.

8 Scheper RJ, Broxterman HJ, Scheffer GL, et al. Overexpression of a $\mathrm{M}(\mathrm{r}) \quad 110,000$ vesicular protein in non-Pglycoprotein-mediated multidrug resistance. Cancer Res 1993;53:1475-9.

9 Scheffer GL, Wijngaard PLJ, Flens MJ, et al. The drug resistance-related protein LRP is the human major vault protein. Nature Med 1995;1:578-82.

10 Kedersha NL, Miquel MC, Bittner D, et al. Vaults II. Ribonucleoprotein structures are highly conserved among nucleoprotein structures are highly conserved among
higher and lower eukaryotes. F Cell Biol 1990;110:895-901.

11 Rome LH, Kedersha NL, Chugani DC. Unlocking vaults: Rome LH, Kedersha NL, Chugani DC. Unlocking vaults:
organelles in search of a function. Trends Cell Biol organelles in

12 Chugani DC, Rome LH, Kedersha NL. Evidence that vault ribonucleoprotein particles localize to the nuclear pore complex. F Cell Sci 1993;106:23-9.

13 Kickhoefer VA, Rajavel KS, Scheffer GL, et al. Vaults are up-regulated in multidrug-resistant cell lines. $\mathcal{F}$ Biol Chem Mol Biol 1998;273:8971-4

14 Izquierdo MA, Scheffer GL, Flens MJ, et al. Broad distribution of the multidrug resistance-related vault lung resistance protein in normal human tissues and tumors. $A m \mathcal{F}$ Pathol 1996;148:877-87.

15 Cordon-Cardo C, O'Brien JP, Casals D, et al. Multidrugresistance gene (P-glycoprotein) is expressed by endothelial cells at blood-brain barrier sites. Proc Natl Acad Sci USA 1989;86:695-8.

16 Flens MJ, Zaman GJR, van der Valk P, et al. Tissue distribution of the multidrug resistance associated protein (MRP). Am f Pathol 1996;148:1237-47.
17 Thiebaut F, Tsuruo $\mathrm{T}$, Hamada $\mathrm{H}$, et al. Cellular localization of the multidrug-resistance gene product P-glycoprotein in normal human tissues. Proc Natl Acad Sci USA 1987;84:7735-8.

18 van der Valk P, van Kalken CK, Ketelaars H, et al. Distribution of multi-drug resistance associated P-glycoprotein in normal and neoplastic human tissues. Ann Oncol 1990;1: $56-64$.

19 Ikeguchi M, Shirai H, Kaibara N, et al. Expression of $\mathrm{P}$-glycoprotein in the normal colorectal epithelium. Int $\mathcal{f}$ Oncol 1995;7:319-24.

20 Scheper RJ, Bulte JW, Brakkee JG, et al. Monoclonal antibody JSB-1 detects a highly conserved epitope on the P-glycoprotein associated with multi-drug-resistance. Int $\mathcal{F}$ Cancer 1988;42:389-94.

21 Arceci RJ, Stieglitz K, Bras J, et al. Monoclonal antibody to an external epitope of the human mdr1 P-glycoprotein. Cancer Res 1993;53:310-17.

22 Flens MJ, Izquierdo MA, Scheffer GL, et al. Immunochemical detection of the multidrug-resistance-associated protein MRP in human multidrug-resistant tumor cells by monoclonal antibodies. Cancer Res 1994;54:4557-63.

23 Schroeijers AB, Scheffer GL, Flens MJ, et al. Immunohistochemical detection of the lung resistance-related protein (LRP). Am F Pathol 1997;152:373-8.

24 Riddell RH. The adenoma-carcinoma sequence. Prog Clin Biol Res 1988;279:23-33.

25 Nagengast FM, Grubben MJ, van Munster IP. Role of bile acids in colorectal carcinogenesis. Eur F Cancer 1995;31A 1067-70.

26 Hatch FT, Knize MG, Healy SK, et al. Cooked-food mutagen reference list and index. Env Mol Mutagenesis 1988;12: $1-85$

27 Van Tassell RL, Kingston DG, Wilkins TD. Metabolism of dietary genotoxins by the human colonic microflora; the fecapentaenes and heterocyclic amines. Mutat Res 1990; 238:209-21.

28 Penny JI, Campbell FC. Does P-glycoprotein mediate the efflux of transport of dietary carcinogen in normal human intestine? [Abstract] Gastroenterology 1995;108:A525.

29 Lown KS, Kolars JC, Ghosh M, et al. Induction of MDR1 expression in normal rat and human intestine in vivo [abstract]. Gastroenterology 1996;109:A344.

30 Kool M, de Haas M, Scheffer GL, et al. Analysis of expression of cMOAT (MRP2), MRP3, MRP4, MRP5, homologs of the multidrug resistance-associated protein gene (MRP1), in human cancer cell lines. Cancer Res 1997;57: 3537-47.

31 Evers R, Zaman GJR, van Deemter L, et al. Basolateral localization and export activity of the human multidrug resistance-associated protein in polarized pig kidney cells. $\mathcal{F}$ Clin Invest 1996;97:1211-18.

32 Paulusma CC, Bosma PJ, Zaman GJR, et al. Congenital jaundice in rats with a mutation in a multidrug resistanceassociated protein gene. Science 1996;271:1126-8.

33 Jedlitschky G, Leier I, Bucholz U, et al. ATP-dependent transport of gluthathione S-conjugates by the multidrug resistance-associated protein. Cancer Res 1994;54:4833-6.

34 Müller M, Meijer C, Zaman GJR, et al. Overexpression of the gene encoding the multidrug resistance-associated protein results in increased ATP-dependent gluthathione S-conjugate transport. Proc Natl Acad Sci USA 1994;91: 13003-37.

35 Keppler D, Leir I, Jedlitschtey G. Transport of glutathione conjugates and glucoronides by the multidrug resistance proteins MRP1 and MRP2. Biol Chem 1997;278:787-91.

36 Loe DW, Almquist KC, Deeley RG, et al. Multidrug resistance protein (MRP-) mediated transport of leukotriene $\mathrm{C} 4$ and chemotherapeutic agents in membrane vesicles: demonstration of glutathione-dependent vincristine transport. F Biol Chem 1996;271:9675-82.

37 Herbergs J, de Bruine AP, Marx PTJ, et al. Chromosome abberations in adenomas of the colon. Proof of trisomy 7 in tumour cells by combined interphase cytogenetics and immunocytochemistry. Int f Cancer 1994;57:781-5.

38 Slovak ML, Ho JP, Cole SPC, et al. The LRP gene encoding a major vault protein associated with drug resistance maps proximal to MRP on chromosome 16: evidence that chromosome breakage plays a key role in in MRP or LRP gene amplification. Cancer Res 1995;55:4214-19.

39 Meijer GA, Hermsen MAJA, Baak JPA, et al. Progression from colorectal adenoma to carcinoma is associated with non-random chromosomal gains as detected by comparative genomic hybridization. $\mathcal{F}$ Clin Pathol 1998;51:901-10.

40 Nooter K, Bosman FT, Burger H, et al. Expression of the multidrug resistance-associated protein (MRP) gene in primary non-small-cell lung cancer. Ann Oncol 1996;7:7581.

41 Konishi F, Morson BC. Pathology of colorectal adenomas: a colonoscopic survey. F Clin Pathol 1982;35:830-41.

42 Vargas PA, Alberts DS. Primary prevention of colorectal cancer through dietary modification. Cancer 1992;70: 1229-35. 